\title{
La fabrication de l'alumine à Gardanne en Provence et les risques technologiques : à la recherche du point d'équilibre?
}

\author{
The plant of alumina at Gardanne in Provence and the technological \\ risks: in search of the point of equilibrium?
}

\author{
Philippe Mioche ${ }^{1}$ \\ ${ }^{1}$ Université Aix-Marseille, laboratoire TELEMME 7303, France, miochephilippe@yahoo.com
}

Le directeur de l'usine d'alumine de Gardanne lors d'une réunion du comité d'établissement en 2006. En France, "La pression est mise sur l'environnement vis-à-vis de l'industrie lourde. On aura de plus en plus de problèmes pour survivre au niveau environnemental, il faut être clair. A terme l'industrie lourde n'existera plus que dans les pays en voie de développement, et certainement plus en France. C'est clair et malheureusement définitif; on ne luttera pas contre le courant ${ }^{1}$.

RÉSUMÉ. L'usine d'alumine de Gardanne, dans le sud de la France, est la plus ancienne du monde dans cette industrie. Cette usine très polluante est à l'origine d'une controverse sur le maintien ou l'arrêt de son activité. La question est importante, car ses impacts sur la santé et l'environnement sont importants, elle s'inscrit aussi dans un processus de désindustrialisation de l'Europe. L'objectif de cet article est de retracer les grandes phases de l'histoire de cet établissement pour discuter de l'histoire des risques industriels. La fermeture de cet établissement n'arrêtera pas la production d'alumine dans le monde, aussi le véritable enjeu réside-t-il dans le développement de nouvelles technologies plus respectueuses de l'environnement.

ABSTRACT. The Gardanne alumina plant, in southern France, is the oldest in the world in this industry. This very polluting plant is currently under scrutiny and at the heart of a debate as to whether it should be shut down. This in an important issue because of the significant impact its activity is having on human health and the environment. Further still, the context of this question is framed by the process of deindustrialization currently taking place in Europe. The objective of this article is to trace the major stages in the history of this plant and to discuss the history of industrial risk as a whole. Shutting down this plant alone will not stop the global production of alumina, so the real challenge lies in developing new environmental technologies.

MOTS-CLÉS. processus de Bayer, alumine, résidus, boue rouge.

KEY-WORDS. Bayer process, alumina, residues, red mud.

\section{Introduction}

L'usine d'alumine de Gardanne, la plus ancienne du monde dans cette industrie, est l'objet en 2016 d'une controverse, sinon d'une polémique d'ampleur internationale à propos des risques technologiques que ses émissions de nuisances et de résidus provoquent dans l'environnement régional et dans la Méditerranée. L'enjeu est le maintien ou l'arrêt de cette activité et il s'est instauré une course contre la montre entre la mise en œuvre de nouveaux dispositifs environnementaux et les demandes d'interruption de la production. La question est sociétale avec des enjeux pour l'environnement et pour la santé, et elle s'inscrit dans le processus en cours de la désindustrialisation de l'Europe.

Nous proposons de retracer les grandes lignes de l'histoire de cet établissement puis de concentrer le propos sur l'histoire des risques technologiques avant de conclure à propos de la controverse. 
La documentation est abondante sur cet établissement grâce notamment aux recherches réalisées depuis 30 ans dans le cadre de l'Institut pour l'histoire de l'aluminium (IHA) ${ }^{2}$. Cette contribution prend aussi appui sur les archives de l'usine qui ont été déposées aux Archives départementales ${ }^{3}$ ainsi que sur de nombreux témoignages oraux que nous avons recueillis de puis la période du centième anniversaire de l'usine en 1983.

\section{L'effacement des fondamentaux historiques de la plus ancienne usine du monde}

La localisation de l'usine en 2016 repose sur les données d'origine en 1893 et celles-ci sont devenues des éléments de sa fragilité contemporaine. A quelques centaines de mètres de la gare de la ligne ferroviaire Marseille-Aix et du centre de la ville qui était une bourgade de 3000 Provençaux à la fin du $19^{\mathrm{e}}$ siècle devenue une ville de 20000 habitants ${ }^{4}$. Elle est là du fait de la proximité de l'ancienne exploitation de lignite nécessaire à sa consommation d'énergie. Par une ancienne voie secondaire (Gardanne-Carnoules), elle était reliée aux gisements de bauxite du Var et par le réseau Paris-LyonMéditerranée au port de Marseille ainsi qu'aux usines d'électrolyse de l'alumine dans les Alpes. La soude nécessaire à la mise en œuvre du procédé Bayer de fabrication de l'alumine provenait de l'usine Solvay de Salin de Giraud en Camargue. La main-d'œuvre était abondante, affluant de l'Italie de la misère et des différents vagues migratoires à Marseille ${ }^{5}$.

La production d'alumine continue en 2016, mais toutes ces causes initiales de l'implantation ont disparu. L'exploitation du lignite a cessé en $2003^{6}$, l'usine quant à elle recourait déjà à d'autres sources d'énergie, le gaz notamment. Les dernières mines de bauxite ont fermé en 1990 à l'issue d'un programme annoncé sur vingt ans ${ }^{7}$. La bauxite traitée à Gardanne provient de Guinée et parfois d'Australie ou du marché international. Elle arrive au port de Fos et elle est acheminée de façon coûteuse à Gardanne par camions ou par trains. La soude, toujours nécessaire, ne vient plus de Camargue où la production a été interrompue en $1961^{8}$, elle est achetée sur le marché.

L'alumine produite était initialement, exclusivement ou presque, à usage métallurgique, c'est-à-dire destinée à l'électrolyse de l'alumine dans les usines alpines et pyrénéennes. Le destin de ce produit était totalement lié à celui de la croissance du métal léger dont la vigueur a longtemps étonné l'histoire industrielle ${ }^{9}$. La dynamique de l'aluminium se poursuit de nos jours, mais elle se réalise ailleurs, en

2. Fondé en 1986, l'Institut pour l'histoire de l'aluminium (IHA) met son savoir-faire et ses collections au service de l'histoire de l'aluminium et de la préservation de son patrimoine http://www.histalu.org/; l'Institut est aussi la cheville ouvrière de l'iconothèque et de la bibliothèque de l'aluminium (http://www.culturalu.org/fr/accueil.php) où on trouvera de nombreuses archives numérisées et des photographies de toutes les époques; y compris à propos de l'usine de Gardanne. Nous exprimons notre reconnaissance à l'égard de cet institut et de ses personnels.

3. Dassé P., Piquet J., « Les archives de l'exploitation de la bauxite et des usines de fabrication de l'alumine d'Aluminium Pechiney: sauvegarde, traitement et accessibilité », Provence Historique, tome LXV, fascicule 258, juillet décembre 2015, pp. 317-332.

4. Mioche P., L'alumine à Gardanne de 1893 à nos jours. Une traversée industrielle en Provence. Grenoble, Presses Universitaires de Grenoble, 1994.

5. Lopez R., Témime E., Migrance. Histoire des migrations à Marseille. Tome 2 : L'expansion marseillaise et «l'invasion italienne » (1830-1918), Aix, Edisud, 1990.

6. Daumalin X., Domenichino J., Mioche P., Raveux O., Gueules noires de Provence. Le bassin minier des Bouches-du-Rhône (1744 - 2003), Marseille, Editions Jeanne Laffitte, 2005.

7. Arnaud C., Guillon J.-M., Les gueules rouges, un siècle de bauxite dans le Var, Centre Départemental de Documentation Pédagogique du Var, 1989. Lesclous R., «What bauxite strategy? An overview of the different players and their behaviour from 1890 to the present», Cahiers d'Histoire de l'Aluminium, n40, p. 11-30, 2008. Grinberg I., Laparra M., Mioche P., «What bauxite strategy did French aluminium producers adopt? From the pretence of a monopoly to lost opportunities (1890 - 2000), Cahiers d'Histoire de l'Aluminium, Journal for the History of Aluminium, n 55, décembre 2015.

8. Daumalin X., Lambert O., Mioche P., Un aventure industrielle en Camargue: histoire de l'établissement Solvay de Salin-deGiraud, de 1895 à nos jours, Aix-en-Provence, RF2C éditions, 2011.

9. Grinberg I., Aluminium. Un si léger métal, Découvertes Gallimard, Paris 2003.

(c) 2016 ISTE OpenScience - Published by ISTE Ltd. London, UK - openscience.fr 
Chine notamment, et elle est stable en Europe. Les usines d'aluminium des Pyrénées et des Alpes ont été fermées à l'exception de celle de Saint-Jean-de-Maurienne, sauvée in extremis en 2014, et celle de Dunkerque qui utilise de l'alumine importée. C'est pourquoi la production de Gardanne, tout en se maintenant en volume, s'est progressivement recentrée vers les alumines techniques dans les années 1980, puis vers les alumines de spécialité plus récemment. Cette alumine, de plus haute valeur ajoutée que l'alumine métallurgique, est livrée à des utilisateurs très variés et, parmi eux, des fabricants de produits des nouvelles technologies de communication comme les téléphones ou les tablettes.

L'histoire managériale de l'usine va dans le même sens. Elle a longtemps été abritée dans de grandes entreprises et son isolement juridique est aujourd'hui croissant. Deux ans après sa création, l'entreprise initiale, la Société française de l'alumine pure, est achetée par PCAC (Produits chimiques d'Alais et de Camargue) qui devient le pivot du futur groupe Pechiney en 1921. C'est ainsi que pendant 82 ans Gardanne est un établissement Pechiney, dans sa division alumine, considérée alors comme un segment de la fabrication de l'aluminium. Mais en 2003, suite à l'endettement du groupe et à des initiatives malencontreuses du management français, l'ex-champion national de l'aluminium fait l'objet d'un rachat hostile par le canadien Alcan ${ }^{10}$. Celui-ci est à son tour racheté par le groupe australo-britannique Rio-Tinto en 2007 dans le cadre d'une mondialisation amplifiée de l'aluminium. L'histoire ne s'arrête pas là car les activités d'alumines de Rio-Tinto, soit trois usines de transformation de l'alumine qui fabriquent du corindon (La Bathie, 160 salariés; Beyrède, 100 salariés) et Alufin (Teutschenthal, Allemagne, 50 salariés) ainsi que la production d'alumines de spécialités de Gardanne sont vendues à un fonds d'investissements diversifiés en 2012, HIG Capital, qui constitue l'entreprise Altéo pour gérer ces quatre établissements. Ultime rebondissement au cours de l'été 2016, la presse évoque des discussions pour la vente des trois usines de transformation à l'industriel Imérys, héritier d'Imétal. L'établissement de Gardanne deviendrait ainsi l'unique site de la société Altéo. Pour son président, Frédéric Ramé : «Cela permettrait à Altéo, dont la production d'alumines de spécialité à Gardanne est le cour de métier, de se concentrer sur son projet d'entreprise ${ }^{11}$.

L'usine de Gardanne a toujours été fidèle au procédé Bayer à l'exception de la période de la Première Guerre mondiale où elle a tenté vainement un autre procédé. De point de vue de l'histoire technique, l'usine de Gardanne est particulièrement riche. On évoquera pour mémoire le fait que deux des «pères fondateurs » de l'industrie mondiale de l'aluminium, Karl Bayer et Paul Héroult, sont venus en personne se pencher sur le berceau de Gardanne entre 1893 et 1905. En vérité, les mises au point ont été nombreuses pour une production de quelques centaines de tonnes au début du $20^{\mathrm{e}}$ siècle. L'usine de Gardanne devient ensuite la référence technique du Bayer pour les différentes usines d'alumine de Pechiney et elle accède à un rayonnement mondial pour certains procédés qu'elle met au point. Cette dynamique est produite par le regroupement des activités de recherche bauxite et alumine à Gardanne au cours de la Seconde Guerre mondiale ${ }^{12}$. Parmi les résultats les plus importants de ces recherches, on doit mentionner «l'attaque continue» du procédé Bayer sans interruption dans les années 1950 qui permet à l'usine des gains de productivité et de production prodigieux. Elle atteint, à partir des années 1960, le niveau de capacités de production qui reste celui de nos jours: environ 500000 tonnes par an. Ce capital de savoir-faire techniques engage l'établissement dans des expériences de diffusion technologique et d'ingénierie et conduit certains salariés à contribuer personnellement à la construction ou à la rénovation d'usines comme en Guinée, en Grèce ou dans l'ex-Yougoslavie. Pechiney, qui disposait par ailleurs d'une maîtrise reconnue dans les techniques des

10. Thaure P., Pechiney?... Vendu !, Paris, Presses des mines ParisTech, 2007.

11. La Provence, 30 juillet 2016 , p. 7.

12. Morel P., (dir), Histoire technique de la production d'aluminium, Presses Universitaires de Grenoble, Grenoble, 1991, Le Roux M., Un siècle de recherche industrielle à Pechiney, Paris, Éditions Rive droite,

1998. Carbonell M., Grunberg I., Laparra M., Le blanc et le noir. Cinquante ans de recherches sur la production d'aluminium au $L R F$, IHA/RF2C, 2012. 
cuves d'électrolyse pour l'aluminium, était alors en compétition avec l'autre grande entreprise de l'innovation de l'alumine, l'américain Alcoa. On retiendra en tout cas que sous le couvert d'une dénomination pérenne, «le Bayer», le procédé en question a été développé par une multitude d'innovations continues et qu'il n'a plus grand-chose à voir avec le procédé initial, à part les principes physiques et chimiques fondamentaux ${ }^{13}$. Incontestablement, Gardanne a été un haut lieu de l'innovation de l'industrie de l'alumine ${ }^{14}$. Toutefois, les fonctions de recherche de l'usine ont été progressivement dissoutes dans les groupes internationaux, Alcan puis Rio Tinto, au cours des années 2000. En 2016, les activités d'innovation, qui sont restées à Rio-Tino sans passer à Altéo, se concentrent sur les procédés d'ingénierie du transport de l'alumine.

L'environnement social de l'usine a beaucoup changé. Outre la croissance démographique de la ville et de l'agglomération aixoise, la sociologie du territoire est en mutation. Le prix de l'immobilier est en forte augmentation. Cette commune a été marquée par l'importance de ses populations étrangères et ouvrières dans les années trente. Les natifs hors de France y étaient alors majoritaires. Les mines, qui occupaient jusqu'à 2000 emplois pendant la Seconde Guerre, ont fermé en 2003. La centrale thermique, réalisée dans l'après-guerre, connait bien des péripéties actuellement. L'emploi est une préoccupation communale même si l'essor remarquable de la micro-électronique dans la zone proche de Rousset Peynier depuis les années 1990 absorbe en partie les entrants sur le marché du travail, y compris les enfants de mineurs ou de l'usine d'alumine ${ }^{15}$. Celle-ci conserve un volant d'emploi d'environ 400 personnes en salariés directs. Elle est un des éléments de continuité du territoire avec le maire communiste, Roger Meï, réélu depuis 1977. En matière sociale, un changement important est venu des méthodes de recrutement dans les années 2000. Les nouveaux managers, suivant les orientations générales des politiques de ressources humaines, et soucieux de ne pas laisser perdurer des dynasties de salariés, ont rompu avec la tradition Pechiney d'embauches privilégiées des enfants de salariés. Il s'agit aussi d'accompagner l'élévation des qualifications de l'industrie, en particulier dans le contexte du passage des alumines métallurgiques aux alumines de spécialité qui appelle des qualifications spécifiques. En conséquence, on observe une déconnexion entre les salariés et le territoire proche. Les recrutés récents viennent de l'aire métropolitaine Aix-Marseille et, de facto, ils sont moins attachés à la vie quotidienne de Gardanne qu'à l'époque où Pechiney offrait la piscine et les colonies de vacances ${ }^{16}$. Enfin, comme ailleurs dans l'industrie, les managers récents ont externalisé de nombreuses fonctions de l'usine, du nettoyage au gardiennage, et bien d'autres encore. S'ajoute à cela un recours important aux intérimaires qui ne font que passer par définition.

Au total, l'usine dont l'immense silhouette rouge jaillit encore à l'entrée de la ville est comme la butte témoin d'une industrialisation ancienne. Pour autant, l'Histoire n'est pas immobile et le management, engagé dans la niche porteuse des alumines de spécialité, a modifié bien des habitudes anciennes, il a obtenu des résultats importants en matière de sécurité technologique et progressé dans la limitation des nuisances environnementales.

\section{Les progrès en matière de sécurité technologiques et de respect de l'environnement}

La fabrication de l'alumine, comme celle de l'aluminium, a toujours été une activité dangereuse et polluante. La dangerosité de l'industrie de l'alumine repose notamment sur la mise en œuvre de la

13. Mioche P., «Contribution à l'histoire du procédé Bayer. Le procédé à Gardanne 1893-2012 », in Dominique Barjot et Marco Bertilorenzi (dirs), Aluminium du métal de luxe au métal de masse (XIXème-XXIème siècle). From Precious metal to mass commodity (19th-21 st century), Paris, Presses Universitaires de Paris Sorbonne, 2014.

14. Morlock J.-Y., « Les innovations technologiques majeures dans le procédé Bayer depuis les origines », in Grinberg I., Griset P., Le Roux M. (dir.), Cent ans d'innovation dans l'industrie de l'aluminium, Paris, l'Harmattan, 1997, pp. 87-108.

15. Carbonell M., Lambert O., Mioche P., De la mine à la puce. Le pôle industriel de la Haute Vallée de l'Arc des origines à nos jours, Mirabeau, RF2C éditions, 2011.

16. Cf. Vindt G., Les Hommes de l'aluminium, histoire sociale de Pechiney, Paris, L'Atelier, 2006.

(c) 2016 ISTE OpenScience - Published by ISTE Ltd. London, UK - openscience.fr

Page $\mid 4$ 
soude dans le procédé Bayer et sur les accidents de pression des machines. Ces dangers sont accrus dans une usine de grande taille où la sédimentation des investissements accentue la complexité des installations et augmente les difficultés de la circulation interne à l'usine. Le 16 juillet 1898, à 3 heures du matin, l'une des deux chaudières Babcock de l'usine explose. Un ouvrier est tué et cinq autres blessés. Sauf silence des archives, ce sont les premières victimes de l'alumine à Gardanne. Entre 1922 et 1928 , le nombre d'accidents du travail avec « incapacité temporaire ou permanente » est supérieur au nombre moyen des différentes usines du groupe. L'année 1924 est marquée par 430 accidents. Le pire est à venir, à 8 heures 30 du matin, le 31 mai 1932. L'équipe en poste a fait démarrer l'opération d'attaque depuis trois quarts d'heure. L'autoclave semble fonctionner normalement, sa pression est d'environ quatre kilos. Quand brusquement la partie supérieure de se déchire, arrachant les rivets. Sous l'effet de la pression, la partie inférieure s'enfonce dans le sol pendant que le haut, soit environ quatre tonnes de métal, part à 80 mètres de hauteur, arrachant la toiture et détruisant une dizaine d'autoclaves voisins. La pièce métallique retombe à 350 mètres de son point de départ, dans des terrains appartenant alors à la mine. L'accident provoque la mort de sept personnes et blesse dix-huit autres. A l'image de l'usine qui emploie de nombreux immigrés, il y avait un Arménien, un Espagnol, deux Français et trois Italiens. Cet accident est le plus grave jamais survenu à Gardanne.

L'attention croissante accordée aux questions de sécurité permet une diminution progressive des accidents du travail. Le taux d'accident s'élève à $16 \%$ en 1952, puis il oscille autour de $7 \%$ entre 1953 et 1959 avant de chuter à moins de $2 \%$ en 1962. Plus tard encore, le directeur de l'usine, Mme Dominique Simon, célèbre en 2000 le cap des 1000 jours sans accident avec arrêt de travail. Mais le recours croissant à des personnels extérieurs limite les progrès en matière de sécurité. En janvier 2009, sur 14 accidents, 12 ont concerné des salariés d'entreprises extérieures. Comme l'affirme un membre du comité d'entreprise : "Nous ne serons jamais une usine non dangereuse. Il y a de la soude; de la température; des flammes; de la pression. Il y a du danger partout. L'idée est d'être suffisamment vigilant pour minimiser au maximum le risque ». Au demeurant, la période Alcan de l'usine (2003-2007) introduit de nouvelles consignes de sécurité qui ne sont pas remises en cause par Rio-Tinto ${ }^{17}$. Cette victoire pour la sécurité n'est pas un cas isolé et doit être portée au crédit de l'industrie manufacturière.

La pollution, interne à l'usine et extérieure, comporte des formes différentes : bruits, émanation de poussières de bauxite, de particules dans l'air, enfin et surtout, production de résidus. Les résidus ou boues rouges ${ }^{18}$ sont une question très importante pour l'histoire industrielle et pour l'histoire environnementale globale car ils constituent un des plus importants déchets industriels en volume de la planète. A ce jour, et depuis les débuts de l'alumine-aluminium industriel, on estime les quantités déversées à 3 milliards de tonnes. Ce volume augmente d'environ 120 millions de tonnes par an ${ }^{19}$. L'histoire et le devenir des résidus de Gardanne renvoient à des interrogations planétaires.

Entre 1893 et 1906, les boues rouges sont déversées à Gardanne sur un terrain à proximité de la voie ferrée. Dès son démarrage, l'usine est confrontée à des protestations du voisinage. M. Montmaney est propriétaire d'une maison voisine. Il porte plainte en 1899 pour la «contamination de son puits ». Il est indemnisé mais il n'acceptera de vendre son bien qu'en 1921. Ce cas est un exemple parmi bien d'autres que les recherches à venir exhumeront. Cet exemple dessine les contours d'un compromis durable entre les industriels et les populations avoisinantes. Quand l'industriel est confronté à une plainte, il propose une indemnisation. Quand la plainte émane d'un périmètre proche, l'industriel

17. Mioche P., «L'usine d'alumine de Gardanne, du local au global, 1893-2016. La mondialisation continue ?», Actes du Colloque de Chicoutimi (FRALUBEC), à paraître.

18 Soudan P., Historique technique et économique de la fabrication de l'alumine, document dactylographié, Paris, IHA, 1970, p. 26. «Le terme 'boues rouges' a été universellement adopté pour les résidus, riches en oxyde de fer, provenant de l'attaque de la bauxite ».

19. Bauxite Residue Management: Best practice, World Aluminium, juillet 2015. En ligne.

(c) 2016 ISTE OpenScience - Published by ISTE Ltd. London, UK - openscience.fr 
propose le rachat. A Salindres dans le Gard, il y a en 1876135 procès en cours au tribunal d'Alès à propos des fumées de l'usine. Pour en finir, H. Merle fait acheter les terrains des plaignants. Ceux-ci déploient une stratégie d'offre en espérant le meilleur prix. On retrouve cette forme de régulation partout et elle se prolonge jusqu'aux années 1970, au moins en Europe.

Pour l'usine de Gardanne, l'augmentation de la production sature le site de stockage initial. L'usine envisage de transporter les boues dans le Var, près des mines de bauxite, puis le directeur, Alfred Guénivet, s'oriente vers les vallons d'Encorse à Bouc-Bel-Air. La ville est disposée à vendre à raison de 1000 F. l'hectare. Le directeur entame les discussions avec les Eaux et Forêts en 1902 et obtient l'autorisation de déverser ses résidus. Un transporteur aérien, le «téléphérique », est mis en service en 1906 et pendant soixante ans, la question de l'évacuation des résidus est «provisoirement » réglée. La régulation par plaintes et indemnisations se perpétue discrètement devant les tribunaux. En 1960, le téléphérique a été remplacé par des conduites qui se déversent dans 7 bassins des vallons de MangeGarri et d'Encorse. Les réservoirs sont pleins et ne peuvent plus accepter de boues rouges d'autant que l'industriel est confronté au risque sismique. C'est alors que l'entreprise prépare la mise en place du «sea line ${ }^{20}$.

A la lumière des débats de 2016, les controverses de la période 1963-1966 montrent la diversité des postures et l'apprentissage environnemental que Pechiney a connu. Constatons que le débat politique régional est complexe car le principal partisan de la conduite est le maire de Gardanne, le socialiste Victor Savine (1891 - 1978), maire de 1929 à 1941 et de 1945 à 1977. Il est président du Conseil général des Bouches-du-Rhône de 1964 à 1967. Le principal adversaire de la conduite est le maire socialiste de Cassis, Emmanuel Agostini (1890-1974), maire de 1945 à 1971. Ces différentes postures sont arbitrées par le maire socialiste de Marseille, Gaston Deferre. L'affaire n'est pas discrète. A l'impact médiatique d'Alain Bombard et de Paul Ricard, Pechiney riposte par une campagne d'information avec l'aide de l'agence Havas. Il y a une manifestation de 1000 personnes dans les rues de Cassis en septembre 1965. Pechiney distribue la brochure «Alumine et Méditerranée » à 6500 exemplaires. En août 1968, le Monde donne quitus à Pechiney: "Il semble donc que toutes les précautions aient bien été prises et que la marge de sécurité choisie soit bien supérieure à celle qui existe dans d'autres pays. [...] Aussi peut-on se demander si finalement, en mettant en garde les touristes et les habitants contre un danger probablement imaginaire, le comité de défense ne fait pas de la contre-publicité à sa région. Son action n'aura cependant pas été inutile, dans la mesure où elle a obligé l'administration et l'industrie à étudier avec plus de précision le problème posé $»^{21}$.

Peu de temps après, Pechiney prend une autre décision qui met sérieusement en cause l'environnement régional : l'ouverture de la mine de bauxite des Canonettes aux Baux de Provence. L'entreprise se heurte à nouveau à une forte mobilisation, notamment de la Ligue des Alpilles ${ }^{22}$. Pechiney renonce à l'exploitation du minerai dans cet espace très touristique au début des années 1990 .

Par ailleurs, le 8 juillet 1974, déversement accidentel d'aluminate de soude dans le ruisseau de la Molx à Gardanne provoque une enquête administrative ${ }^{23}$. Si Pechiney a fait preuve dans les années soixante d'une insuffisante prise en compte de l'environnement et de l'opinion publique, l'entreprise a

20. Nous avons publié en 2011 une contribution sur l'histoire du sea line qui reste accessible en ligne : Mioche P., Alumine et risques industriels : le cas des boues rouges et des résidus, Article en ligne, 2011, http://www.ohm-provence.org/animations.html et http://www.histalu.org/

21. « Les boues rouges à la mer », Lavallard J.-L., le Monde, 19 août 1965.

22. Mioche P., «Pechiney face au NIMBY des Alpilles », Industries en Provence. Dynamiques d'hier et d'aujourd'hui, n¹1, décembre 2003, pp. 25 et suivantes.

23. Ministère de l'Agriculture, Service régional de l'aménagement des eaux. Principaux rejets industriels. Bassin versant de la Luyne et de ses affluents, janvier 1981.

() 2016 ISTE OpenScience - Published by ISTE Ltd. London, UK - openscience.fr

Page | 6 
fait son apprentissage dans les années $1970^{24}$. Un des paradoxes de la crise actuelle, est que Pechiney Ugine Kuhlmann faisait figure de pionnier en ayant signé en 1975 avec les pouvoirs publics un accord de réduction volontaire de la pollution ${ }^{25}$. Y compris du point de vue du dialogue avec l'opinion publique, la comparaison de la gestion des résidus à Gardanne et de celle de l'usine d'alumine de Porto-Vesme en Sardaigne tourne à l'avantage de la première ${ }^{26}$.

\section{La controverse environnementale}

Depuis la conférence de Barcelone en 1995, il est convenu que l'arrêt des versements des résidus dans la fosse de Cassis s'interromprait le 31 décembre 2015. Cette décision n'a pas été contestée et elle est mise en œuvre depuis cette date. Il n'y a plus de «boues rouges» déversées en Méditerranée ${ }^{27}$. Cependant, Altéo a demandé l'autorisation de déverser les résidus liquides de la fabrication en poursuivant l'utilisation du sea-line. Cette demande a fait l'objet de nombreux rapports et elle demeure au centre de la controverse principale. A ce jour, l'entreprise a obtenu une autorisation préfectorale pour déverser les résidus liquides jusqu'en 2021. D'autres débats environnementaux portent sur la qualité de l'air, la qualité de l'eau dans la zone d'épandage de Bouc-Bel-Air et son éventuelle radioactivité. La controverse devient polémique en septembre 2016 et certains médias évoquent «un chantage à l'emploi ».

Plusieurs éléments de court terme et de longue durée se superposent et conduisent l'usine de Gardanne est au centre d'un débat international. L'écoulement des boues rouges de l'usine d'alumine d'Ajka en Hongrie en 2010 a saisi l'opinion. "L'événement [d'Ajka] a notamment réactivé les négociations auparavant atténuées pour qualifier ces boues émanant de l'usine, autrement dit pour faire exister et disparaître les débordements auxquels donne lieu l'usine de Gardanne ${ }^{28}$. Cet événement spécifique s'inscrit aussi dans un contexte de désindustrialisation de plus en plus perceptible en Europe ${ }^{29}$, "l'industrie des années 1970 est entrée dans l'ère du soupçon " ${ }^{30}$, et l'acceptabilité de l'industrie et de ses nuisances régresse dans l'opinion. La tension est forte entre les désirs pour l'emploi et les aspirations à une protection toujours plus étendue de l'environnement et de la santé. C'est peut-être un moment à passer en attendant que s'épanouisse la troisième industrialisation avec l'économie du numérique et la digitalisation des entreprises? «A moins d'admettre simplement que la révolution industrielle se poursuit imperturbablement autour de nous. Qu'elle ne cesse d'être, comme je le pense avec admiration mais pas toujours avec plaisir, en mouvement ${ }^{31}$. Mais ces tensions sont fortes et se traduisent par des questions pour l'usine de Gardanne.

24. Boullet D., «La gestion de l'environnement dans les entreprises industrielles en France : une mise en perspective historique (1950-1990) », Entreprises et histoire 4/2006 (n 45), p. 54-73.

Loizon M.-C., Pezet A., «L'entreprise verte et les boues rouges. Les pratiques controversées de la responsabilité sociétale de l'usine d'alumine de Gardanne. 1960-1966», Entreprises et Histoire, n²5, 2006/4.

25. Massard-Guilbaud G., «Environnement : une bien lente prise de conscience », in Dictionnaire du Patronat, Jean-Claude Daumas (dir), Paris, Flammarion, pp.995-1000, 2010.

26. Mioche P. et Bertilorenzi M., « Les résidus de l'alumine à Portovesne (Italie) et à Gardanne/Cassis (France), des années soixante à nos jours », in Laura CENTERINI et Xavier DAUMALIN (dir.), Pollutions industrielles et espaces méditerranéens XVIII-XXI siècle, Karthala -Maison Méditerranéenne des sciences de l'homme, Aix-en-Provence-Paris, p. 275-299, 2015.

27. L'usine grecque de Distomon qui utilisait un «sea line » a arrêté ses versements en 2012. Cf. Grinberg I., Mioche P., Aluminium de Grèce, l'usine aux trois rivages, Grenoble, Presses Universitaires de Grenoble, 1996.

28. Letté M., «Le tournant environnemental de la société industrielle au prisme d'une histoire des débordements et de leurs conflits », Vingtième Siècle. Revue d'histoire 1/2012 (n 113), p. 142-154.

29. Daumas J.-C., Kharaba I., Mioche P., La désindustrialisation, une fatalité ? Presses Universitaires de Bourgogne, à paraître.

30. Woronoff D., Histoire de l'industrie en France. Du XVIe siècle à nos jours, Paris, Seuil, 1994, p. 592.

31. Braudel F., L'identité de la France, Paris, Arthaud, tome 2. 1986. 
Que faire des boues rouges? Il faut souligner que l'utilisation d'un sous-produit de l'industrie chimique est un principe de base de cette industrie, aussi ancien qu'elle. "La suppression d'une pollution passe souvent par la valorisation économique du rejet polluant ${ }^{32}$. Depuis les débuts de la fabrication à Gardanne, l'entreprise a reçu des propositions dans ce sens. En mai 1895, le directeur des "Mines de Tréfys », près d'Avignon, propose à $P$. Héroult un «traité » pour récupérer les résidus provenant de la bauxite, "auquel cas, nous pourrions immédiatement entrer en campagne pour trouver des débouchés » ${ }^{33}$. Le directeur de Gardanne, A. Guénivet, négocie avec les Hauts-Fourneaux de Saint-Louis à Marseille afin de vendre les boues rouges à l'entreprise sidérurgique ${ }^{34}$. Mais les briquettes se délitent à cause de la forte présence de soude et ne conviennent pas. La piste de la réutilisation du fer contenu dans la bauxite a suscité de nombreux espoirs de réutilisation. Le dialogue technique s'est poursuivi longtemps avec les sidérurgistes. Au début des années 1960 encore, à l'occasion des projets d'acier à Fos, Gardanne songe à vendre ses boues rouges et leur fer. Mais pour résumer, les sidérurgistes ne veulent pas de ce fer d'une qualité inférieure à leurs besoins et à leur fabrication d'aciers à haute valeur ajoutée. D'une façon générale, et depuis fort longtemps, les producteurs d'aluminium et d'alumine ont envisagé dans leurs programmes de recherche un nombre considérable de réutilisations totales ou partielles des résidus. L'industrie de l'aluminium a aussi mis au point un procédé de fabrication d'alumine sans bauxite, à partir de terres argileuses, le $\mathrm{H}^{35}$. C'est au Centre de recherche de l'alumine de Gardanne que Pechiney depuis 1963 cherchait à trouver un nouveau procédé pour la production d'alumine à partir de minerais autres que la bauxite. Le «procédé $\mathrm{H} »$, basé sur la décomposition des matières premières par voie acide (d'où le « $\mathrm{H} »$ ), est passé successivement du stade « laboratoire », au « grand laboratoire» dans la décennie qui a suivi. En 1974, il semble sur le point de pouvoir déboucher sur un véritable procédé industriel et il est rebaptisé « $\mathrm{H}+$ » pour souligner les améliorations effectuées depuis les intuitions originelles de 1963. Pechiney propose alors une association 50/50 sur les coûts passés et futurs avec le canadien ALCAN. Le consortium franco-canadien lance le programme secret de recherche, le "procédé $\mathrm{H}+$ », afin de construire un « atelier expérimental » (ATEX), où les capacités de production sont d'environ 20 t/jour d'alumine.

La nouvelle usine pilote est construite entre juillet 1975 et mai 1976 à l'Estaque. Une cinquantaine de jeunes opérateurs sont recrutés pour un contrat de trois ans ${ }^{36}$. Avec l'ATEX, le procédé $\mathrm{H}+$ marche et il est prometteur: on peut fabriquer de l'alumine à partir des terres argileuses de façon économiquement rentable sous réserve d'ajustements concernant la technologie, la consommation d'énergie et la gestion des résidus du nouveau procédé. Cependant, l'affaiblissement du cartel des producteurs de bauxite et la découverte de nouvelles réserves de bauxite dans des pays considérés " sûrs », comme l'Australie, conduit à interrompre l'exploitation de ce projet en dépit des résultats obtenus. Le point d'équilibre économique n'existe plus mais la solution technologique est prête. Les détracteurs d'Altéo en 2016 font grand cas du procédé Orbite mis au point au Canada ${ }^{37}$. Selon les ingénieurs consultés par nous, celui-ci s'inspire largement du H+.

L'usine d'alumine de Gardanne a cessé de verser les résidus solides en décembre 2015. Il n'y a plus de boues rouges en Méditerranée. Dans le monde, la technique du lagunage («lagooning») est généralement adoptée par les producteurs d'alumine. Cette méthode fonctionne d'autant mieux dans les environnements peu peuplés, l'alcalinité des boues est d'autant réduite quand on peut les neutraliser avec de l'eau de mer et elle fonctionne mieux dans les climats chauds où l'évaporation est facilitée.

32. Massard-Guilbaud G., op. cit, 2010.

33. Charbon des mines de Tréfys à P. Héroult, 6 mi 1895. Archives de La Praz à Saint-Jean-de-Maurienne.

34. Raveux O., «Un demi-siècle de sidérurgie provençale : les Hauts-Fourneaux de Saint-Louis (1855-1905)», Industries en Provence. Dynamiques d'hier et d'aujourd'hui, n 3, mars, pp. 18-27, 1999.

35. Bertilorenzi M., Mioche P., «Between strategy and diplomacy. A long run appraisal of alumina alternative technologies, 1900s1970s», Cahiers d'histoire de l'aluminium, n. 51, p p. 43- 63, 2014.

36. Entretien avec Christian Mérono, mars 2012.

37. http://www.orbitetech.com/French/technologie/le-procede-d-orbite/default.aspx

(c) 2016 ISTE OpenScience - Published by ISTE Ltd. London, UK - openscience.fr 
Gardanne a entrepris un ambitieux programme de valorisation des résidus. En 1994, l'usine a déposé la marque «bauxaline $(\mathcal{C}$ » et elle a réalisé des investissements dans la construction de filtre-presse en 2007. Indépendamment de la question des effluents liquides, la question de la vente de la bauxaline est incontournable à court et à moyen terme. Car les boues préparées et séchées ne peuvent être stockées indéfiniment. Cela fait longtemps que l'usine espère valoriser ses résidus, on comprend bien pourquoi. Ce n'est sans doute pas une question de prix de revient, on peut même imaginer que l'entreprise serait heureuse de les valoriser à prix négatifs ou subventionnés. Mais les usages tardent à se multiplier et l'avenir est incertain car les raffineries d'alumine développent les procédés de séchage des boues.

Que faire du Bayer? Il y a bien longtemps que dans l'usine la question est posée de raffiner de l'alumine importée et de fermer la partie rouge au profit d'un maintien et d'un éventuel développement de la partie blanche. «La question avait été posée à Monsieur Occello [ancien directeur] et il avait fait la bonne réponse, il avait dit "si on ferme le Bayer à Gardanne, on ferme l'usine ». Le Directeur : Ce n'est pas ce que je dis. Pour l'instant, nous avons intérêt à garder le Bayer, mais avec toutes les alumineries qui se construisent on risque de trouver des gens avec qui on pourrait s'entendre pour un contrat à long terme d'hydrate international à partir duquel on pourrait continuer à vivre. La mort du Bayer ne sera pas forcément la mort de l'usine. Aujourd'hui on a un avantage à vivre avec le Bayer, même s'il est l'un des plus chers du monde, parce qu'il nous permet d'avoir 2 qualités d'alumines. Que le Bayer soit fragile, c'est clair. Peut-être qu'il fermera un jour ; à force de l'annoncer, il y a bien un moment où ça arrivera! ${ }^{38}$. On peut raffiner de l'alumine sans disposer d'un Bayer, c'est le cas à l'usine Martinswerk de Bergheim (près de Cologne en Allemagne). Cette évolution, qui est assez peu évoquée en public poserait d'autres problèmes. Cela supposerait une réduction grosso mode de moitié de l'espace occupé par l'usine et vraisemblablement de l'emploi. Cela n'interviendrait pas dans les coûts de réhabilitation des sols libérés. Le modèle économique issu d'une telle évolution est incertain car l'entreprise ne bénéficierait plus des taxations avantageuses pour les produits importés sous forme brute.

Que faire de l'usine? On évitera la discussion, récurrente et hautement improbable de la création d'une nouvelle unité d'alumine dans la région (sur le littoral de Fos, qui peut y croire ?). Le différentiel des coûts de production est considérable dans le marché globalisé de l'alumine. En 2010, il s'élève à 180 US\$ la tonne en Australie, contre 202 en Chine, et 328 en Italie ${ }^{39}$. Si une nouvelle raffinerie d'alumine devait être construite (par qui ?), il est peu vraisemblable de penser qu'elle pourrait l'être dans l'Union Européenne.

Dans l'Union Européenne, il reste 6 raffineries d'alumine, dont deux en Allemagne. La plus importante d'entre elles - deux millions de tonnes d'alumine métallurgique, soit 4 fois la production de Gardanne - est l'usine d'Aughinish, en Irlande. Elle appartient à la multinationale russe, RUSAL. La France connaît un net déficit pour les alumines métallurgiques. En 2012 et 2013, le solde commercial est déficitaire de 300 à 400000 tonnes d'alumine. Les importations viennent à $65 \%$ d'Irlande, c'est-àdire de l'usine Russal. Le prix de l'alumine exportée par la France est environ le double de celle qui est importée (soit $0.54 €$ le kilo en 2012 et 0.60 en 2013 contre $0.32 €$ et 0.31$)^{40}$. La fragile rentabilité de l'usine de Gardanne repose sur le fait que les investissements y sont largement amortis. La conjoncture est nettement favorable pour les alumines de spécialités et d'une certaine façon, chaque journée gagnée par l'usine est une journée bien gagnée.

38 Réunion du Comité d'Etablissement, 24 Mai 2007.

39 «Les coûts d'exploitation de l'alumine dans le monde », Université du Québec à Chicoutimi, juin 2010. L'usine italienne est à l'arrêt en 2016.

40 J. F. Lalbé, BRGM, octobre 2014. http://docplayer.fr/10038627-Tableau-1-statistiques-francaises-d-import-export-de-produitsbruts-et-intermediaires-d-aluminium.html 
La question clairement posée par les détracteurs d'Altéo est celle de sa fermeture. On citera l'exemple d'une question posée au Sénat le $1^{\text {er }}$ avril $2016^{41}$ : «Je suis sensible à la question de l'emploi, mais, sous ce rapport, il faut songer aussi aux pêcheurs et à tous les métiers de la mer! L'environnement et la santé publique doivent-ils constituer les variables d'ajustement de la préservation de l'emploi ? N'aurait-il pas mieux valu engager une reconversion, plutôt que de prolonger artificiellement la vie d'un site industriel dont Mme la ministre de l'environnement ellemême a souligné la vétusté ? Comment faire respecter la protection de l'environnement dans le parc national des Calanques?».

Si l'usine ferme, le coût du désinvestissement et de la dépollution des sols en centre urbain sera considérable.

Si l'usine ferme, les besoins du marché en alumines de spécialité ne disparaîtront pas car le marché est porteur. Les produits seront fabriqués ailleurs, en Afrique ou en Amérique Latine, où l'environnement et la santé sont moins protégés que dans l'Union Européenne. Ne serait-il pas plus cohérent de rechercher encore les solutions techniques et économiques afin de trouver un nouveau point d'équilibre entre la production de biens et la préservation de l'environnement et de la santé plutôt que de déplacer le problème qu'une partie de l'opinion européenne ne veut plus voir ?

Ne convient-il pas de maîtriser nos inquiétudes en matière d'environnement et de santé au profit d'une mobilisation du dialogue entre sciences, techniques, production et population ? Faut-il s'engager vers d'autres horizons que la production de biens manufacturés? Revendiquer un avenir où les emplois des nouveaux entrants reposeraient sur le tourisme et les services aux personnes dépendantes?

La plus ancienne usine d'alumine du monde est menacée de fermeture. Le propos n'est pas ici de prendre sa défense mais d'inviter à explorer jusqu'au bout le dialogue entre sciences, techniques, industries et citoyens. Sachant que l'arrêt de Gardanne ne ralentira pas la demande et la production d'alumines de spécialités dans le monde, le véritable enjeu global est d'améliorer les techniques de gestion des résidus de trouver de nouvelles solutions les moins agressives possible. Rien ne permet d'exclure qu'un nouveau point d'équilibre ne puisse être trouvé et les producteurs s'y emploient activement sous forme de programmes internationaux de recherche soutenus par l'Union européenne.

41. Question d'actualité au gouvernement $n^{\circ} 0806 \mathrm{G}$ de Mme Mireille Jouve (Bouches-du-Rhône - RDSE), publiée dans le JO Sénat du 01/04/2016. 